\title{
Improvement of cytotoxicity of mitoxantrone and daunorubicin by candidone, tephrosin, and bavachinin
}

\author{
Sina Darzi ${ }^{1,2}$. Seyed Abbas Mirzaei ${ }^{1} \cdot$ Fatemeh Elahian $^{1} \cdot$ Amir Peymani $^{3} \cdot$ Babak Rahmani $^{4}$. \\ Shaghayegh Pishkhan Dibazar ${ }^{5} \cdot$ Sadegh Shirian $^{6,7} \cdot$ Leila Shakeri Chaleshtori $^{8} \cdot$ Ehsan Aali ${ }^{3}$
}

Received: 5 November 2020 / Accepted: 6 September 2021

(C) The Author(s), under exclusive licence to Springer Nature B.V. 2021

\begin{abstract}
Background Flavonoids have been demonstrated to have the ability of sensitizing cancer cells to chemotherapy and inverse multidrug resistance via various mechanisms, such as modulating of pumps. The therapeutic effect of candidone, tephrosin, and bavachinin in treatment of cancer, particularly to overcome multidrug resistance (MDR) is largely unknown. The capacity of these agents in sensitization of MDR cells is investigated in the current work.

Methods and results We analyzed the impact of candidone, tephrosin, and bavachinin, as chemosensitizer on cell cytotoxicity, $P$ - $g p$ and $A B C G 2$ mRNA expression level on two multidrug resistant cells, ABCG2 overexpressing human epithelial breast cancer cell line (MCF7/MX), and P-gp overexpressing human gastric adenocarcinoma cell line (EPG85.257RDB). The inhibitory concentration of 50\% (IC50) of daunorubicin in EPG85.257RDB cells in combination with IC10 of Bavachinin, Tephrosin, and Candidone were $6159 \pm 948,4186 \pm 665,730 \pm 258 \mathrm{nM}$, and this data in MCF7/MX cell were 1773 \pm 534 , $7160 \pm 405$ and $3340 \pm 622 \mathrm{nM}$ respectively. These three flavonoids dose-dependently decreased the viability of MCF7/ MX and EPG85.257RDB and significantly $(\mathrm{p}<0.05)$ decreased IC50 of daunorubicin and mitoxantrone except Tephrosin in MCF7/MX cells. Candidone and Bavachinin were the most potent chemosensitizer in EPG85.257RDB and MCF7/MX cells respectively. Flavonoids did not reduce mRNA expression of P-gp and ABCG2 after $72 \mathrm{~h}$ treatment, except Candidone in EPG85.257RDB and Bavachinin in MCF7/MX cells.

Conclusions This effect is not time-dependent, and flavonoids have their own patterns that are cell-dependent. In general, tephrosin, candidone, and bavachinin had the potential of sensitizing MDR cells to mitoxantrone and daunorubicin.
\end{abstract}

Keywords MDR1 ABCG2 - Flavonoid $\cdot$ Multidrug resistant $\cdot$ Real-time pcr

Ehsan Aali

en.aali@gmail.com; e.aali@qums.ac.ir

Sina Darzi

S.darzi@qums.ac.ir

Seyed Abbas Mirzaei

mirzaei.a@skums.ac.ir

Fatemeh Elahian

elahian.f@skums.ac.ir

Amir Peymani

apeymani@qums.ac.ir

Babak Rahmani

brahmani@qums.ac.ir

Sadegh Shirian

shirian85@gmail.com
2 Health Product Safety Research Center, Qazvin University of Medical Sciences, Qazvin, Iran

3 Medical Microbiology Research Center, Qazvin University of Medical Sciences, Qazvin, Iran

4 Department of Molecular Medicine, Qazvin University of Medical Sciences, Qazvin, Iran

5 Department of Biotechnology, Qazvin University of Medical Sciences, Qazvin, Iran

6 Department of Pathology, School of Veterinary Medicine, Shahrekord University, Shahrekord, Iran

7 Shiraz Molecular Research Center, Dr. Daneshbod Lab, Shiraz, Iran

8 Department of Biology, Faculty of Basic Sciences, Payame Noor University (PNU), Isfahan, Iran

1 Department of Molecular Medicine, Faculty of Advanced Technologies, Shahrekord University of Medical Sciences, Shahrekord, Iran 